\title{
Use of a neuromuscular stimulator to increase anal sphincter pressure
}

\author{
LW Riedy*,1,3, R Chintam ${ }^{2,3}$ and JS Walter ${ }^{1,4}$ \\ ${ }^{1}$ Rehabilitation R\&D Section, Hines VA Hospital, Hines, Illinois, IL 60141, USA; ${ }^{2}$ Spinal Cord Injury Service, Hines \\ VA Hospital, Hines, Illinois, IL 60141, USA; ${ }^{3}$ Department of Internal Medicine, Loyola Medical Center, Stritch \\ School of Medicine, Maywood, Illinois, IL 60153, USA; ${ }^{4}$ Department of Urology, Loyola Medical Center, Stritch \\ School of Medicine, Maywood, Illinois, IL 60153, USA
}

\begin{abstract}
Objectives: The objective of this study was to determine if short periods of electrical stimulation with perianal electrodes could increase anal pressures.

Material and methods: Anorectal responses to electrical stimulation were evaluated in five healthy SCI patients. Anorectal pressures were recorded with a small pressure-recording balloon before, during, and immediately following stimulation. A battery-powered stimulator with self-adhering surface electrodes, two inches in diameter was used. Stimulating parameters consisted of $300 \mu$ s pulse duration, $35 \mathrm{~Hz}$ stimulating frequency. A current response study was conducted by using short periods of electrical stimulating with currents from $0-100 \mathrm{~mA}$ until a maximal pressure was recorded. Each current setting was conducted for $13.2 \pm 9.7 \mathrm{~s}$ before increasing to the next higher current, and fatigue was reduced by including a 5 -minute rest between stimulations.

Results: Four of the five subjects had strong anal contractions with perianal stimulation. Increases in pressure ranged from 38 to $125 \mathrm{~cm} \mathrm{H}_{2} \mathrm{O}$ based on maximal responses at currents ranges of 60 to $100 \mathrm{ma}$. Even during the short periods of stimulation used here, fatigue was apparent. There was an average drop of $11 \%$ in anal pressure over the $13 \mathrm{~s}$ of stimulation. Rectal pressures were unchanged with perianal stimulation.

Conclusions: Perianal stimulation with surface electrodes is an approach that might be considered in the future for management of fecal incontinence in individuals with spinal cord injury. Further studies are needed to assess the feasibility of using chronic perianal surface electrical stimulation to sustain anal sphincter contractions.
\end{abstract}

Spinal Cord (2000) 38, 724-727

Keywords: Neuroprosthetics; electric stimulation; spinal cord injury; fecal incontinence

\section{Introduction}

The site of the most frequently documented gastrointestinal problems for chronic spinal cord injury (SCI) patients is the colon. ${ }^{1}$ The majority of SCI patients experience constipation while a smaller subset experience fecal incontinence. In this subset, an injury to S2 S3 nerve roots leads to a loss of conscious control of the external anal sphincter resulting in fecal incontinence. ${ }^{1}$ In addition, other SCI patients experiencing constipation are at risk of developing fecal incontinence secondary to a prolonged use of suppositories, enemas and manual evacuation techniques. Medical concerns include dermatological problems such as rashes and stool contamination and infection of sacral decubitus ulcers. $^{2}$ In addition to these medical problems, bowel incontinence is viewed as a social embarrassment, often leading to periods of isolation and low self-esteem. ${ }^{2-4}$

Rehabilitation of the gastrointestinal tract is an important part of patient care following SCI. Once

*Correspondence: LW Riedy, VA Hines Hospital, Rehabilitation R\&D Section (151), Hines, Illinois, IL 60141, USA paralytic ileus resolves, patients are generally placed on a bowel training program with the overall goal of evacuating the bowel regularly and thereby minimizing episodes of fecal incontinence. To assist in evacuating the bowels, high-fiber diets, bulking agents, stool softeners and laxatives are often used. To reduce episodes of fecal incontinence associated with outlet obstruction or overflow incontinence, patients manually remove hardened stool from the rectum prior to initiating reflex defecation. Even with a well-established bowel program, accidents occur. Depending on the level and severity of the injury, some patients experience difficulty cleaning up after an accident. Combined with the prevalence of decubitus sacral ulcers in this patient population, fecal incontinence is a serious concern. Thus, there is a need for improved care.

In general, various treatments have been used to manage bowel incontinence. ${ }^{2,4-5}$ In non-SCI patients, treatments have included biofeedback, an incontinence training program, surgery and electrical stimulation. Of particular interest to this study have been the 
applications involving intra-anal plug electrodes. ${ }^{6-8}$ transrectal probes, ${ }^{9}$ and sacral spinal nerve electrodes. ${ }^{10}$ In non-SCI patients, Hopkins and Lightwood ${ }^{6}$ evaluated custom fabricated intra-anal plug electrodes in nine patients with a variety of gastrointestinal symptoms in addition to fecal incontinence. Improvements were noted in four patients, however, follow-up was limited. In addition to patients reporting difficulty retaining the plug and difficulty in urinating with the plug in place, there was one report of an anal burn associated with the electrode. Glen ${ }^{7}$ evaluated commercially available intra-anal plugs in 30 nonSCI patients for urinary or fecal incontinence. Of these 30 patients, nine suffered from fecal incontinence. Five patients were able to control their fecal incontinence with the plug and one patient was reported as being cured. Treatments varied from 3 weeks to 8 months and the follow-up was not indicated for most of the patients. Pescatori et $a l^{8}$ used anal plug electrodes and reported improvement in two-thirds of the non-SCI patients with varying causes of incontinence. None of the patients, however, became fully continent. Based on anal manometry, increases in squeeze pressure were noted suggesting that the stimulation positively reinforced the voluntary contractile activity of the striated sphincter. In a group of myelomeningocele patients, a patient group similar to SCI, a transrectal probe was evaluated. ${ }^{9}$ Approximately one-third of the children were reported as complete successes with no further need to wear diapers for stool incontinence after completing the program.

The mechanisms, through which these types of electrical stimulation function to elicit the anal reflex can include voluntary augmentation of the external anal sphincter, direct activation of the pudendal nerve motor pathways and reflex activation through the afferent neurons. ${ }^{8,11-13}$ After a complete SCI, any conscious or centrally mediated contribution to anal sphincter contraction is lost. Perianal electrical stimulation can generate both the motor and reflex pathways sufficiently to induce anal contractions. ${ }^{13}$ Therefore, the efficacy of surface electrical stimulation to manage fecal incontinence in patients with traumatic SCI warrants further investigation. We evaluated a neuromuscular stimulator with moderatesize surface area electrodes adjacent to the anal sphincter in patients with SCI. Only acute currentresponse stimulation studies were conducted in these investigations.

\section{Materials and methods}

\section{Patients}

Five healthy male subjects were recruited through physician recommendations. Informed consent was obtained from all participants prior to the study. The protocol was approved by the Hines VA Human Studies Subcommittee. Inclusion criteria consisted of male and female traumatic SCI patients, aged 19 years and older who had concerns about their bowel program. Exclusion criteria consisted of patients with active urine infection, decubitus ulcers, cardiovascular disease, and cardiac pacemakers/defibrillators. Although females were included, there were no females with SCI available to participate.

\section{Anorectal manometry}

Anorectal manometry was carried out at the bedside just prior to, during and after perianal stimulation. A two-channel chart recorder (Servigor model 102, Norma Goerz Instruments, Elk Grove Village, IL, USA) was used to record pressure. Continuously variable gain was accomplished with two $10 \mathrm{~K} \Omega \quad 10$ turn potentiometers. The chart recorder was powered by a $12 \mathrm{~V}$ rechargeable battery.

A medically approved sterile, water filled, balloon catheter (Rusch Inc., Duluth, GA, USA) was attached to the strip chart recorded via a pressure transducer (Model PNEU05, World Precision Instruments, Inc. Sarasota, FL, USA). The exciter voltage was supplied with a $5 \mathrm{~V}$ regulated supply consisting of a $9 \mathrm{~V}$ battery powering a 7805 voltage regulator with a LED indicator and on/off switch. The transducer was fed directly to the chart recorder that had sufficient gain and offset adjustments for this signal. Calibration was accomplished with a water column.

The dimensions of the flaccid balloon were $5 \mathrm{~cm}$ in length and $2 \mathrm{~cm}$ in width. The balloon was initially placed into the rectum for recording rectal pressure before, during and after stimulation. The balloon was then pulled into the anal canal for anal pressure recordings before, during and after stimulation.

\section{Perianal stimulation}

Stimulation was conducted with a commercially available, small battery powered stimulator (Neuromuscular II, Medical Devices, Inc., Saint Paul, MN, USA) and self adhering surface electrodes (Uni-patch Encore $^{\mathrm{TM}}$ Plus, Wabash, MN, USA). The surface electrodes were composed of 2 inch diameter round patches coated with a self-adhering gel. For perianal stimulation, two surface electrodes were placed externally adjacent to the anal opening along the midline. The separation between the electrodes was approximately 1 inch across the anal verge. In all cases, the skin was clean of any feces at the time of evaluation and did not require any further preparation.

Stimulation was conducted with $300 \mu$ s charge balanced pulses at up to $100 \mathrm{~mA}$ with a compliance voltage of $100 \mathrm{~V}$. All stimulation was below this compliance voltage. Additional parameters included 35 pps stimulating frequency, 1-s ramp up time, and 0.25 -s ramp down time. Short stimulation periods were used at each stimulation current and 5 min rest was used between stimulations to reduce the effects of fatigue. Current response studies were conducted with the current increased for each stimulation until a 
maximal pressure was reached. Maximum current is that which a further increase in current does not result in a further increase in pressure. Stimulation was for $13.2 \pm 9.7 \mathrm{~s}$.

\section{Results}

As shown in Table 1, the age range was from 48 to 70 years, with a mean age of 56 years. The spinal cord injuries consisted of two cervical and three thoracic. All of the injuries were classified as complete based on the ASIA scale. The average number of years postinjury was 17.6, with a low of 6 and a high of 30 years. Gastrointestinal complaints ranged from fecal incontinence to constipation with two patients reporting rectal pain.

Effects of different procedures were determined with the pressure recording balloon in the rectum. Coughing, and abdominal pressure increased the rectal pressure whereas perianal stimulation did not. In addition, none of these interventions had an effect on spontaneous rectal activity (number of contractions/min or amplitude). The balloon was then pulled into the anal canal.

Increases in anal pressures were recorded in four (subjects 1-3,5) out of five subjects with stimulation. Anal pressure responses to different currents are shown in Figure 1. Maximal currents for the four patients that responded to stimulation ranged between 60 and $100 \mathrm{~mA}$. For patient $1,100 \mathrm{~mA}$ resulted in a higher pressure than lower currents of $70-80 \mathrm{~mA}$. However, a second stimulation at $100 \mathrm{~mA}$ resulted in a maximal pressure similar to that at $70-80 \mathrm{~mA}$. Therefore, for this patient it was not clear whether 70 or $100 \mathrm{~mA}$ results in the maximal pressure. For patient 2, a maximum stimulating current was not reached. The highest current used was $70 \mathrm{~mA}$. For patient 3, a maximum pressure of $38 \mathrm{~cm} \mathrm{H}_{2} \mathrm{O}$ was obtained for both 60 and $70 \mathrm{~mA}$. Therefore, $60 \mathrm{~mA}$ appears to be a maximal current. For patient 5 , a near maximal current of $85 \mathrm{~mA}$ was obtained as only a small increase in pressure $\left(12 \mathrm{~cm} \mathrm{H}_{2} \mathrm{O}\right)$ was obtained when the current was increased from 70 to $85 \mathrm{~mA}$. None of the subjects reported any discomfort from the stimulation.

Fatigue is an important concern in applying anal stimulation. The increases in anal pressure were not sustained over the length of the applied stimulus and a $11 \%$ drop-off was observed over an average of $13.2 \pm 9.7 \mathrm{~s}$ for the nine stimulations where this could be determined.

\section{Discussion}

Four of our five subjects evaluated had strong contractions in anal pressure with perianal stimulation with the maximal pressure ranging between 38 $100 \mathrm{~mA}$, Table 1. The increases in pressure were not sustained for the duration of the applied stimulus which is consistent with other reports. ${ }^{12}$ Brindley et al ${ }^{12}$ reported almost complete fatigue after $2 \mathrm{~min}$ of stimulation in non-SCI patients using an anal plug electrode. Their findings showed that the anal sphincter fatigue was dependent upon the stimulating frequency. Similarly, we observed anal sphincter fatigue with a $11 \%$ drop-off in anal pressure over a stimulation period of $13.2 \pm 9.7 \mathrm{~s}$. A slower stimulating frequency may reduce the rate of sphincter fatigue. As a means of minimizing sphincter fatigue, various studies have examined the possibility of permanent training or

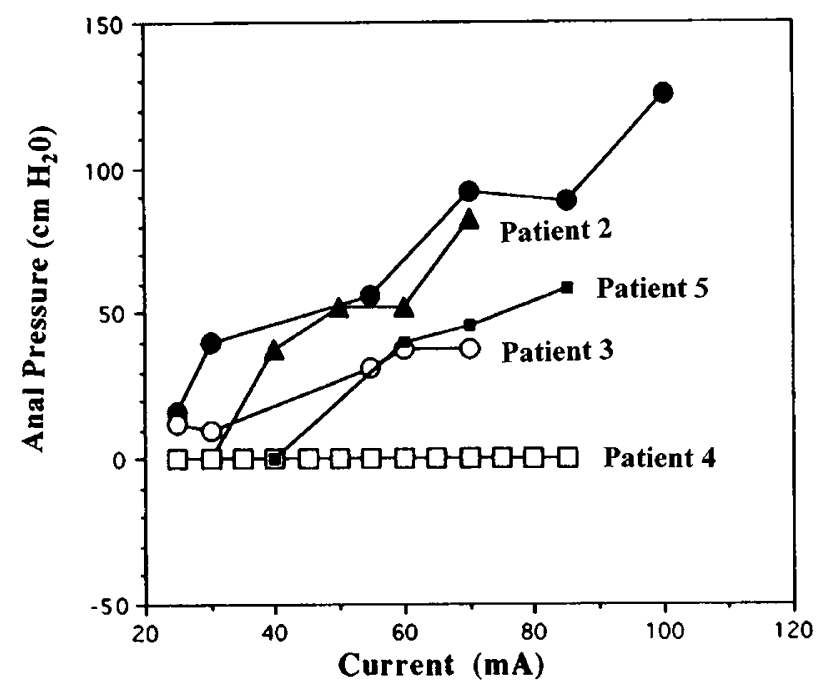

Figure 1 Current response curves using the Neuromuscular II stimulator and Unipatch surface electrodes placed externally adjacent to the anal opening along the midline of

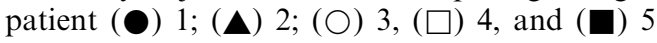

Table 1 Study subjects and anal responses to stimulation

\begin{tabular}{lccccrr}
\hline Patient & Sge & SCI level & Years injured & $\begin{array}{c}\text { Gastro-intestinal } \\
\text { status }^{a}\end{array}$ & $\begin{array}{c}\text { Current (mA) } \\
\text { Ctimulation }\left(\mathrm{cm} \mathrm{H}_{2} \mathrm{O}\right)\end{array}$ \\
\hline 1 & 50 & C4, comp. & 11 & 3 & 100 & 125 \\
2 & 70 & T7, comp. & 6 & 1,2 & 70 & 83 \\
3 & 61 & T10, comp. & 30 & 1,4 & 70 & 38 \\
4 & 51 & T12, comp. & 26 & 2 & 85 & 0 \\
5 & 48 & C8, comp. & 15 & 2 & 85 & 58 \\
\hline
\end{tabular}

${ }^{\mathrm{a}} 1=$ constipation; $2=$ intermittent fecal incontinence; $3=$ rectal pain associated with defecation; $4=$ intermittent rectal pain. For all measurements, the electrodes were placed externally adjacent to the anal opening along the midline 
transforming fast twitch, fatigable type II fibers into slow twitch, fatigue resistant type I fibers. ${ }^{10}$ This conversion could enhance the efficacy of perianal stimulation.

Normally, the internal anal sphincter is largely responsible for maintaining fecal continence. In cases where the internal anal sphincter function is no longer sufficient, the external anal sphincter may be able to take over this function. The primary mechanism through which perianal stimulation results in contraction of the sphincter is through stimulation of the pudendal afferents or nervemotor fibres. To achieve the maximal anal sphincter contraction, the maximal number of pudendal nerve fibers needs to be stimulated. In other studies, pain/sensation can limit the duration of stimulation and the maximal current. $^{7,12}$ As our study evaluated surface electrical stimulation in a group of complete SCI individuals, local pain/sensation was not a limiting factor. We were able to evaluate high current. High anal pressures were recorded in four of five patients. For patient 4, there was no increase in anal pressure over the current range evaluated. It is possible that this patient did not respond to stimulation because of lower motor neuron injury, resulting in diminished pudendal nerve excitability.

As the aim of this study was to assess the feasibility of acute perianal electrical stimulation for increasing anal sphincter pressure following SCI, the long-term effects of electrical stimulation on anal sphincter pressures have yet to be studied. The surface electrodes were easy to place. The patients in this study did not, however, volunteer to use the device for longer periods of time or at home. They did not feel that their occasional episodes of fecal incontinence warranted use of this device. Additionally, patients found the devices cumbersome. However, future evaluation of this technique needs to address sphincter fatigue, conversion of fast twitch to slow twitch muscle fibers, and maximizing the recruitment of motor fibers.

\section{Acknowledgments}

This material is based upon work supported by the Office of Research and Development and the VA Rehabilitation R\&D Service Department of Veterans Affairs (1840PA).

\section{References}

1 Cosman BC, Stone JM, Perkash I. Gastrointestinal complications of chronic spinal cord injury. J Am Paraplegia Soc 1991; 14: $175-181$.

2 Somers MF. Bowel and bladder management. In Spinal Cord Injury: Functional Rehabilitation. Appleton and Lange, A Publishing Division of Prentice Hall. Chapter 17, 1992, p 299 312

3 Hackler RH. A 25 year prospective mortality study in the spinal cord injury patient: Comparison with the long term living paraplegic. J Urol 1977; 117: 486-488.

4 Banwell JG, Creasey GH, Agarwal AM, Mortimer JT. Management of the neurogenic bowel in patients with spinal cord injury. Urolog Clin North Am 1993; 20: 517 - 526.

5 Jorge JMN and Wexner S. Etiology and management of fecal incontinence. Dis Colon Rectum 1993; 36: 77-97.

6 Hopkinson BR, Lightwood R. Electrical treatment for incontinence. Br J Surg 1967; 54: $802-805$.

7 Glen ES. Effective and safe control of incontinence by the intraanal plug electrode. Br J Surg 1971; 58: 249-252.

8 Pescatori M, Pavesio R, Anastasio G, Daini S. Transanal electrostimulation for fecal incontinence: Clinical, psychologic, and manometric prospective study. Dis Colon Rectum 1991; 34: $540-545$.

9 Palmer LS, Richards I, Kaplan WE. Transrectal electrostimulation therapy for neuropathic bowel dysfunction in children with myelomeningocele. J Urol 1996; 157: 1449-1452.

10 Matzel KE, Stadelmaier U, Hohenfellner M, Gall FP. Electrica stimulation of sacral spinal nerves for treatment of faecal incontinence. Lancet 1995; 346: $1124-1127$.

11 Pederson E, Klemar B, Schroder H, Torring J. Anal sphincter responses after perianal electrical stimulation. $J$ Neurol Neurosurg Psych 1982; 45: 770-773.

12 Brindley GS, Rushton DN, Craggs M. The pressure exerted by the external sphincter of the urethra when its motor nerve fibers are stimulated electrically. J Urol 1974; 46: 453-462.

13 Varma JS, Smith AN. Anal reflexes. In Henry MM, Swash M (eds). Coloproctology and the Pelvic Floor. ButterworthHeinemann, 1992, pp 207-219. 